study was aimed to determine the prevalence and risk factors associated with $\mathrm{T}$. vaginalis among asymptomatic undergraduate students at Babcock University, Ilisan-Remo, Ogun State, Nigeria.

Methodology Descriptive cross-sectional study involving 123 female and 123 male students of Babcock University was done from February 2019 to April 2020. The institution's ethical committee approved the study. Information regarding demographics, behavioural and sexual characteristics was obtained from consenting participants using questionnaire. First-void urine samples were collected from each participant for the detection of $\mathrm{T}$. vaginalis using the traditional wet preparation method and TV in-pouch. The data were analyzed by using IBM SPSS version 23.

Result The prevalence of $\mathrm{T}$. vaginalis among the participants was $12.2 \%(30 / 246)$ using TV in-pouch and $8.5 \%(21 / 246)$ using the traditional wet preparation method. This differential rate was statistically significant. The prevalence rate was slightly higher among females $53.3 \%$ (16/30) compared to males $46.7 \%$ (14/30). Excessive alcohol intake and low condom use lost their significance on multivariate analysis while there was no significant association with prior STI. Recent sexual intercourse $(\mathrm{OR}=22.26,95 \% \mathrm{CI}$ : 4.73-104.65), use of hormonal contraceptives $(\mathrm{OR}=0.07$, 95\% CI: $0.006-$ $0.742)$ and internet-based sex seeking behaviour $(\mathrm{OR}=31.17$, 95\% CI: 2.59-375.19) had increased likelihood of T. vaginalis infection on multivariate analysis.

Conclusion The occurrence of T. vaginalis among the asymptomatic population in this study is very high. Associated risk factors identified may be helpful for counseling, screening and management of patient.

\section{P064 EVALUATION OF CHLAMYDIA TRACHOMATIS POSITIVE SPECIMENS ESCAPING DETECTION IN THE APTIMA COMBO 2 ASSAY IN THE UNITED STATES}

${ }^{1} \mathrm{~S}$ Katz ${ }^{*},{ }^{1} \mathrm{D}$ Danavall, ${ }^{1} \mathrm{M}$ Morris, ${ }^{1} \mathrm{~B}$ Herrod, ${ }^{2} \mathrm{M}$ Nye, ${ }^{1} \mathrm{E}$ Kersh, ${ }^{1} \mathrm{R}$ Kirkcaldy, ${ }^{1} \mathrm{~B}$ Raphael. ${ }^{1}$ Centers For Disease Control and Prevention, Atlanta, USA; ${ }^{2}$ Center for Esoteric Testing, Laboratory Corporation of America, Holdings, Burlington, USA

\subsection{6/sextrans-2021-sti.203}

Background The Aptima Combo $2^{\circledR}$ (AC2, Hologic) assay detects Chlamydia trachomatis (CT) and Neisseria gonorrhoeae in clinical specimens. The assay target for CT is a region within the $23 \mathrm{~S}$ ribosomal RNA (rRNA). Recent international reports highlighted a failure to detect CT variants with nucleotide substitutions in the AC2 target region. We explored the presence of CT $23 \mathrm{~S}$ variants in the United States and whether a redesigned AC2 assay would successfully detect them.

Methods A total of 50 specimens (tested using the AC2 assay between December 2019 - February 2020) with CT-negative or equivocal results (and having relative light unit (RLU) signal $\geq 15-99$ ) were selected for further analysis. Additional specimens (RLU <15 [n=300], RLU >100 [n=51]) were also examined. A custom real-time PCR was used to detect CT DNA in these samples and a portion of the CT $23 \mathrm{~S}$ rRNA gene was amplified and sequenced. Samples were then tested using a redesigned AC2 assay provided by the manufacturer as research use only (RUO) reagent.
Results All samples with AC2 results $<15$ RLU were CT-negative by real-time PCR. A subset of five AC2 CT-positive samples with $100+$ RLU were sequenced, which confirmed wild-type $23 \mathrm{~S}$ rRNA sequences. CT DNA was detected in seven of the 50 specimens with $\geq 15-99$ RLU by PCR. Four of these specimens contained wild-type CT 23S rRNA sequences and two separately contained previously unreported 23S rRNA mutations (A1518G, G1526A). The redesigned AC2 assay detected 22 previously CT-negative or equivocal specimens, including six (four wild-type $23 \mathrm{~S}$ rRNA, two variant $23 \mathrm{~S}$ rRNA) of the seven identified by real-time PCR.

Conclusions Our findings show the redesigned AC2 assay is able to detect $23 \mathrm{~S}$ rRNA point mutation variants that were not detected by its predecessor. Neither of these variant strains were associated with recent reports of diagnostic escape mutants in Europe.

\section{P065 QUICK ONLINE STI RISK-ASSESSMENT QUESTIONS FOR ASYMPTOMATIC YOUNG PEOPLE: THE COMPUTER SAID I SHOULD GET TESTED}

${ }^{1} \mathrm{~A}$ Groos ${ }^{*},{ }^{2} \mathrm{~S}$ Peardon-Freeman, ${ }^{2} \mathrm{~K}$ McFarlane, ${ }^{3} \mathrm{~S}$ Braithwaite, ${ }^{4} \mathrm{D}$ Gajjar, ${ }^{1} \mathrm{P}$ Murch, ${ }^{1} \mathrm{C}$ Spucches. ${ }^{1}$ Communicable Diseases Branch, Queensland Health, Herston, Australia; ${ }^{2}$ Health Contact Centre, Health Support Queensland, Brisbane, Australia; ${ }^{3}$ Preventive Health Branch, Queensland Health, Herston, Australia; ${ }^{4}$ Health Service Strategy and Planning, Metro North Hospital and Health Services, Royal Brisbane Hospital, Australia

\subsection{6/sextrans-2021-sti.204}

Background Both healthcare providers and clients identify barriers to sexually transmissible infections (STI) testing associated with time, embarrassment, anxiety or stigma. Online testing options are one mechanism to address some of these constraints and engage people who prefer technology facilitated processes.

Methods Queensland Health in Australia offers a free chlamydia and gonorrhoea urine test and uses four STI risk-assessment questions with yes/no answers to guide client decisions whether to continue to use the online service to order a Webtest. The STI risk-assessment generates high, medium and low-risk categories with messages recommending where or how to get tested. Data was extracted from the Webtest system on 3 August 2020 about the online activity since 3 August 2017 and tests ordered between 3 August 2017 and 30 June 2020.

Results For the high-risk category, the online message recommends testing at a clinic and many people who chose not to order a Webtest $(56.0 \%, 2418 / 4320)$ were in this category. The majority of Webtest orders $(57.2 \%, 3401 / 5943)$ were made from a medium-risk outcome where the online message recommends testing. Very few testing requests are received from people classified as low-risk $(0.3 \%, 20 / 5943)$.

For people with valid STI results $(n=3737)$, the risk-assessment was effective in identifying increased risk. Chlamydia positivity rates were $11.6 \%$ for high $(166 / 1429$, 95\% CI 10.1-13.4), 4.4\% for medium (101/2296, 95\% CI 3.6-5.3), and no positive results received for those with low-risk outcome scores $(0 / 12)$.

Conclusion While not intended as a stand-alone intervention, a personalised internet-based self-assessment tool enabling a 